\title{
Fractional Langevin Equations with Nonseparated Integral Boundary Conditions
}

\author{
Khalid Hilal, Lahcen Ibnelazyz $\mathbb{D}^{\mathbb{D}}$, Karim Guida ${ }^{\mathbb{D}}$, and Said Melliani
}

Sultan Moulay Slimane University, BP 523, 23000 Beni Mellal, Morocco

Correspondence should be addressed to Lahcen Ibnelazyz; ibnelazyzlahcen@gmail.com

Received 17 June 2020; Revised 15 July 2020; Accepted 17 July 2020; Published 19 August 2020

Academic Editor: Laurent Raymond

Copyright (C) 2020 Khalid Hilal et al. This is an open access article distributed under the Creative Commons Attribution License, which permits unrestricted use, distribution, and reproduction in any medium, provided the original work is properly cited.

In this paper, we discuss the existence of solutions for nonlinear fractional Langevin equations with nonseparated type integral boundary conditions. The Banach fixed point theorem and Krasnoselskii fixed point theorem are applied to establish the results. Some examples are provided for the illustration of the main work.

\section{Introduction}

Fractional derivatives give an excellent description of memory and hereditary properties of different processes. Properties of the fractional derivatives make the fractionalorder models more useful and practical than the classical integral-order models.

Several researchers in the recent years have employed the fractional calculus as a way of describing natural phenomena in different fields such as physics, biology, finance, economics, and bioengineering (for more details see [1-9] and many other references).

With the recent outstanding development in fractional differential equations, the Langevin equation has been considered a part of fractional calculus, and thus, important results have been elaborated (see [10-14]).

The Langevin equation was first introduced by Langevin in 1908; it is a fundamental theory of the Brownian motion to describe the evolution of physical phenomena in fluctuating environments $[15,16]$. The fractional model of the Langevin equation as a generalization of the classical one gives a fractional Gaussian process parametrized by two indices, and this fractional model is more flexible for modeling fractal processes $[17,18]$.

The fractional Langevin equation is extensively studied in the literature from both the theoretical and numerical point of views (for more details see [19-25]). In [26], the authors studied a nonlinear Langevin equation involving two frac- tional orders in different intervals. In [27], the authors discussed the existence theory for a nonlinear Langevin equations with nonlocal multipoint and multistrip boundary conditions. In [28], fractional Langevin equations with nonlocal integral boundary conditions have been investigated by Salem et al. In [14], an antiperiodic boundary value problem for the Langevin equation involving two fractional orders has been studied.

Recently, in [29], the authors discussed the nonlinear fractional differential equations with nonseparated type integral boundary conditions; however, the fractional Langevin equations involving nonseparated integral boundary conditions have not been investigated yet; that is why, in this work and motivated by all the works cited above, we study the existence and uniqueness of the fractional Langevin equations with nonseparated integral boundary conditions as follows:

$$
\left\{\begin{array}{l}
{ }^{c} D^{\beta}\left({ }^{c} D^{\alpha}+\lambda\right) x(t)=f\left(t, x(t), I^{p} x(t)\right), \quad t \in[0,1], \\
x(0)+\mu x(1)=\sigma_{1} \int_{0}^{1} g(s, x(s)) d s, \\
{ }^{c} D^{\alpha} x(0)+\mu^{c} D^{\alpha} x(1)=\sigma_{2} \int_{0}^{1} h(s, x(s)) d s, \\
{ }^{c} D^{2 \alpha} x(0)+\mu^{c} D^{2 \alpha} x(1)=\sigma_{3} \int_{0}^{1} k(s, x(s)) d s,
\end{array}\right.
$$


where $0<\alpha<1,1<\beta \leq 2, p>0, \lambda, \mu, \sigma_{1}, \sigma_{2}, \sigma_{3} \in \mathbb{R}^{*}$ with $\mu \neq-1$, and ${ }^{c} D^{\beta},{ }^{c} D^{\alpha}$ are the Caputo fractional derivatives and $f:[0,1] \times \mathbb{R} \times \mathbb{R} \longrightarrow \mathbb{R}$ and $g, h, k:[0,1] \times \mathbb{R} \longrightarrow \mathbb{R}$ are given continuous functions.

This paper is divided into four sections, in which the second provides some notations and basic known results, in the third section, we study the existence and uniqueness of solutions to problem (1), and in the fourth section, we give two examples to illustrate our results.

\section{Preliminaries and Notations}

In this section, we give some notation, definitions, and lemma which are needed throughout this paper.

Definition 1 (see [5]). The fractional integral of order $\alpha>0$ with the lower limit zero for a function $f$ can be defined as

$$
I^{\alpha} f(t)=\frac{1}{\Gamma(\alpha)} \int_{0}^{t}(t-s)^{\alpha-1} f(s) d s
$$

Definition 2 (see [5]). The Caputo derivative of order $\alpha>0$ with the lower limit zero for a function $f$ can be defined as

$$
{ }^{c} D^{\alpha} f=\frac{1}{\Gamma(n-\alpha)} \int_{0}^{t}(t-s)^{n-\alpha-1} f^{(n)}(s) d s,
$$

where $n \in \mathbb{N}, 0 \leq n-1<\alpha<n$, and $t>0$.

Theorem 3 (see [30]). Let $M$ be a bounded, closed, convex, and nonempty subset of a Banach space X. Let $A$ and $B$ be operators such that

(I) $A x+B y \in M$ whenever $x, y \in M$

(II) $A$ is compact and continuous

(III) $B$ is a contraction mapping

Then, there exists $z \in M$ such that $z=A z+B z$.

Lemma 4 (see [5]). Let $\alpha, \beta \geq 0$; then, the following relations hold:

$$
\begin{aligned}
I^{\alpha} t^{\beta} & =\frac{\Gamma(\beta+1)}{\Gamma(\alpha+\beta+1)} t^{\alpha+\beta}, \\
{ }^{c} D^{\alpha} t^{\beta} & =\frac{\Gamma(\beta+1)}{\Gamma(\beta-\alpha+1)} t^{\beta-\alpha} .
\end{aligned}
$$

Lemma 5 (see [5]). Let $n \in \mathbb{N}$ and $n-1<\alpha<n$. If $f$ is a continuous function, then, we have

$$
I^{\alpha c} D^{\alpha} f(t)=f(t)+\alpha_{0}+a_{1} t+a_{2} t^{2}+\cdots+a_{n-1} t^{n-1} .
$$

Lemma 6. Let $y \in C([0,1], \mathbb{R})$. Then, a unique solution of the bondary value problem

$$
\left\{\begin{array}{l}
{ }^{c} D^{\beta}\left({ }^{c} D^{\alpha}+\lambda\right) x(t)=y(t), \quad t \in[0,1], \\
x(0)+\mu x(1)=\sigma_{1} \int_{0}^{1} g(s ; x(s)) d s, \\
{ }^{c} D^{\alpha} x(0)+\mu^{c} D^{\alpha} x(1)=\sigma_{2} \int_{0}^{1} h(s ; x(s)) d s \\
{ }^{c} D^{2 \alpha} x(0)+\mu^{c} D^{2 \alpha} x(1)=\sigma_{3} \int_{0}^{1} k(s ; x(s)) d s
\end{array}\right.
$$

is given by

$$
\begin{aligned}
x(t)= & \frac{1}{\Gamma(\alpha+\beta)} \int_{0}^{t}(t-s)^{\alpha+\beta-1} y(s) d s-\frac{\lambda}{\Gamma(\alpha)} \int_{0}^{t}(t-s)^{\alpha-1} x(s) d s \\
& +A_{1}(t) \int_{0}^{1} h(s ; x(s)) d s+A_{2}(t) \int_{0}^{1} g(s ; x(s)) d s \\
& +A_{3}(t) \int_{0}^{1} k(s ; x(s)) d s+\frac{A_{4}(t)}{\Gamma(\beta-\alpha)} \int_{0}^{1}(1-s)^{\beta-\alpha-1} y(s) d s \\
& +\frac{A_{5}(t)}{\Gamma(\beta)} \int_{0}^{1}(1-s)^{\beta-1} y(s) d s+\frac{\mu \lambda}{(1+\mu) \Gamma(\alpha)} \int_{0}^{1}(1-s)^{\alpha-1} x(s) d s \\
& -\frac{\mu}{(1+\mu) \Gamma(\alpha+\beta)} \int_{0}^{1}(1-s)^{\alpha+\beta-1} y(s) d s,
\end{aligned}
$$

where

$$
\begin{aligned}
A_{1}(t)= & \frac{t^{\alpha} \sigma_{2}(1-\lambda \Gamma(2-\sigma))}{\Gamma(\alpha+1)(1+\mu)}+\frac{t^{\alpha+1} \lambda_{\sigma_{2}} \Gamma(2-\alpha)}{\Gamma(2+\alpha)} \\
& +\left(\frac{\mu}{(1+\mu)^{2} \Gamma(\alpha+1)}-\frac{1}{(1+\mu) \Gamma(\alpha+2)}\right) \Gamma(2-\alpha) \lambda \sigma_{2} \\
& -\frac{\mu \sigma_{2}}{(1+\mu)^{2} \Gamma(\alpha+1)}, \\
A_{2}(t)= & \frac{t^{\alpha} \lambda \sigma_{1}}{\Gamma(\alpha+1)(1+\mu)}-\frac{\sigma_{1}}{1+\mu}+\frac{\mu \lambda \sigma_{1}}{(1+\mu)^{2} \Gamma(\alpha+1)}, \\
A_{3}(t)= & \frac{t^{\alpha} \Gamma(2-\alpha) \sigma_{3}}{\Gamma(\alpha+1)(1+\mu)}+\frac{t^{\alpha+1} \Gamma(2-\alpha) \sigma_{3}}{\Gamma(\alpha+2) \mu}+\frac{\mu \Gamma(2-\alpha) \sigma_{3}}{(1+\mu)^{2} \Gamma(\alpha+1)} \\
& -\frac{\Gamma(2-\alpha) \sigma_{3}}{(1+\mu) \Gamma(\alpha+2)}, \\
A_{4}(t)= & \frac{t^{\alpha} \Gamma(2-\alpha) \mu}{\Gamma(\alpha+1)(1+\mu)}-\frac{t^{\alpha+1} \Gamma(2-\alpha)}{\Gamma(\alpha+2)}+\Gamma(2-\alpha) \\
& \cdot\left(\frac{\mu}{(1+\mu) \Gamma(\alpha+2)}-\frac{\mu}{(1+\mu)^{2} \Gamma(\alpha+1)}\right),
\end{aligned}
$$$$
A_{5}(t)=\frac{t^{\alpha} \mu}{\Gamma(\alpha+1)(1+\mu)}-\frac{\mu^{2}}{(1+\mu)^{2} \Gamma(\alpha+1)}
$$ 
Proof. By applying Lemma 5, we have

$$
\begin{aligned}
\left({ }^{c} D^{\alpha}+\lambda\right) x(t) & =I^{\beta} y(t)+a_{0}+a_{1} t, \\
{ }^{c} D^{a} x(t) & =I^{\beta} y(t)+a 0+a_{1} t-\lambda x(t), \\
x(t) & =I^{\alpha+\beta} y(t)+I^{\alpha} a_{0}+I^{\alpha} a_{1} t-I^{\alpha} \lambda x(t)+a_{2},
\end{aligned}
$$

where $a_{0}, a_{1}, a_{2} \in \mathbb{R}$.

Using the condition ${ }^{c} D^{2 \alpha} x(0)+\mu^{c} D^{2 \alpha} x(1)=\sigma_{3} \int_{0}^{1} k(s ; x$ $(s)) d s$, we obtain

$$
\begin{aligned}
a_{1}= & \Gamma(2-\alpha)\left(\frac { \sigma _ { 3 } } { \mu } \int _ { 0 } ^ { 1 } k \left(s ; x(s) d s+\frac{\lambda \sigma_{2}}{\mu} \int_{0}^{1} h(s ; x(s)) d s\right.\right. \\
& \left.-\frac{1}{\Gamma(\beta-\alpha)} \int_{0}^{1}(1-s)^{\beta-\alpha} y(s) d s\right) .
\end{aligned}
$$

By conditions ${ }^{c} D^{\alpha} x(0)+\mu^{c} D^{\alpha} x(1)=\sigma_{2} \int_{0}^{1} k(s ; x(s)) d s$ and $x(0)+\mu x(1)=\sigma_{1} \int_{0}^{1} g(s ; x(s)) d s$, we have

$$
\begin{aligned}
a_{0}= & \frac{-\Gamma(2-\alpha) \sigma_{3}}{1+\mu} \int_{0}^{1} k(s, x(s)) d s+\frac{(1-\lambda \Gamma(2-\alpha)) \sigma_{2}}{1+\mu} \int_{0}^{1} h(s, x(s)) d s \\
& +d \frac{\lambda \sigma_{1}}{1+\mu} \int_{0}^{1} g(s, x(s)) d s+\frac{\Gamma(2-\alpha) \mu}{(1+\mu) \Gamma(\beta-\alpha)} \int_{0}^{1}(1-s)^{\beta-\alpha-1} y(s) d s \\
& -\frac{\mu}{(1+\mu) \Gamma(\beta)} \int_{0}^{1}(1-s)^{\beta-1} y(s) d s, \\
a_{2}= & \frac{\mu \lambda}{(1+\mu) \Gamma(\alpha)} \int_{0}^{1}(1-s)^{\alpha-1} x(s) d s+\left(\frac{\sigma_{1}}{1+\mu}-\frac{\mu \lambda \sigma_{1}}{\Gamma(\alpha+1)(1+\mu)^{2}}\right) \\
& \cdot \int_{0}^{1} g(s, x(s)) d s-\frac{\mu}{(1+\mu) \Gamma(\alpha+\beta)} \int_{0}^{1}(1-s)^{\alpha+\beta-1} y(s) d s \\
& +\frac{\mu^{2}}{(1+\mu)^{2} \Gamma(\beta) \Gamma(\alpha+1)} \int_{0}^{1}(1-s)^{\beta-1} y(s) d s+\frac{\Gamma(2-\alpha)}{\Gamma(\beta-\alpha)} \\
& \cdot\left(\frac{\mu}{(1+\mu) \Gamma(\alpha+2)}-\frac{\mu^{2}}{(1+\mu)^{2} \Gamma(\alpha+1)}\right) \int_{0}^{1}(1-s)^{\beta-\alpha-1} y(s) d s \\
& +\left[\left(\frac{\mu^{2}}{(1+\mu)^{2} \Gamma(\alpha+1)}-\frac{\mu}{(1+\mu) \Gamma(\alpha+2)} \frac{\Gamma(2-\alpha) \lambda \sigma_{2}}{\mu}\right.\right. \\
& \left.-\frac{\mu \sigma_{2}}{(1+\mu)^{2} \Gamma(\alpha+1)}\right] \int_{0}^{1} h(s, x(s)) d s .
\end{aligned}
$$

Substituting the value of $a_{0}, a_{1}$, and $a_{2}$, we obtain the desired results. And by direct computation, one can obtain the converse of the lemma.

\section{Main Results}

Denote by $X$ the Banach space of all continuous functions from $[0,1] \longrightarrow \mathbb{R}$ endowed with norm $\|x\|=\sup \{|x(t)|$ : $t \in[0,1]\}$.
By Lemma 6, we transform problem (1) into a fixed point problem as $x=P x$, where $P: X \longrightarrow X$ is given by

$$
\begin{aligned}
P x(t)= & \frac{1}{\Gamma(\alpha+\beta)} \int_{0}^{t}(t-s)^{\alpha+\beta-1} f\left(s, x(s), I^{p} x(s)\right) d s \\
& -\frac{\lambda}{\Gamma(\alpha)} \int_{0}^{t}(t-s)^{\alpha-1} x(s) d s+A_{1}(t) \int_{0}^{1} h(s ; x(s)) d s \\
& +A_{2}(t) \int_{0}^{1} g(s ; x(s)) d s+A_{3}(t) \int_{0}^{1} k(s ; x(s)) d s \\
& +\frac{A_{4}(t)}{\Gamma(\beta-\alpha)} \int_{0}^{1}(1-s)^{\beta-\alpha-1} f\left(s, x(s), I^{p} x(s)\right) d s \\
& +\frac{A_{5}(t)}{\Gamma(\beta)} \int_{0}^{1}(1-s)^{\beta-1} f\left(s, x(s), I^{p} x(s)\right) d s \\
& +\frac{\mu \lambda}{(1+\mu) \Gamma(\alpha)} \int_{0}^{1}(1-s)^{\alpha-1} x(s) d s \\
& -\frac{\mu}{(1+\mu) \Gamma(\alpha+\beta)} \int_{0}^{1}(1-s)^{\alpha+\beta-1} f\left(s, x(s), I^{p} x(s)\right) d s .
\end{aligned}
$$

Theorem 2. Suppose that $f:[0,1] \times \mathbb{R}^{2} \longrightarrow \mathbb{R}$ and $h, g$, $k:[0,1] \times \mathbb{R} \longrightarrow \mathbb{R}$ are continuous functions satisfying

$\left(H_{1}\right)$-there exist positive constants $q_{1}, q_{2}$ such that

$$
\left|f\left(t, x_{1}, y_{1}\right)-f\left(t, x_{2}, y_{2}\right)\right| \leq q_{1}\left|x_{1}-x_{2}\right|+q_{2}\left|y_{1}-y_{2}\right| \text {, }
$$

for all $x_{1}, x_{2}, y_{1}, y_{2} \in \mathbb{R}, t \in[0,1]$.

$\left(H_{2}\right)$-there exist positive constants $q_{3}, q_{4}, q_{5}$ such that

$$
\begin{aligned}
& |g(t, x)-g(t, y)| \leq q_{3}|x-y|,|h(t, x)-h(t, y)| \leq q_{4}|x-y|, \\
& |k(t, x)-k(t, y)| \leq q_{5}|x-y|, \quad \forall x, y \in \mathbb{R} .
\end{aligned}
$$

Then there exist a unique solution for boundary value problem (1) provided that $r_{1}<1$, where

$$
\begin{aligned}
r_{1}= & {\left[( q _ { 1 } + \frac { q _ { 2 } } { \Gamma ( p + 1 ) } ) \left(\frac{1}{\Gamma(\alpha+\beta+1)}+\frac{A_{4}}{\Gamma(\beta-\alpha+1)}\right.\right.} \\
& \left.+\frac{A_{5}}{\Gamma(\beta+1)}+\left|\frac{\mu}{(1+\mu) \Gamma(\alpha+\beta+1)}\right|\right)+\frac{|\lambda|}{\Gamma(\alpha+1)} \\
& \left.+\left|\frac{\mu \lambda}{(1+\mu) \Gamma(\alpha+1)}\right|+A_{1} q_{4}+A_{2} q_{3}+A_{3} q_{5}\right]
\end{aligned}
$$

and $A_{i}=\max _{t \in[0,1]}\left|A_{i}(t)\right|$ for $i=1,2, . ., 5$.

Proof. We set $\sup _{0 \leq t \leq 1}|f(t, 0,0)|=M_{0}, \quad \sup _{0 \leq t \leq 1}|g(t, 0)|=M_{1}$, $\sup _{0 \leq t \leq 1}|h(t, 0)|=M_{2}, \sup _{0 \leq t \leq 1}|k(t, 0)|=M_{3}$. 
Let $B_{r}=\{x \in X:\|x\| \leq r\}$ the ball with radius $r$, where $r \geq\left(r_{2} /\left(1-r_{1}\right)\right)$, with

$$
\begin{aligned}
r_{2}= & \frac{M_{0}}{\Gamma(\alpha+\beta+1)}+\frac{M_{0} A_{4}}{\Gamma(\beta-\alpha+1)}+A_{1} M_{1}+A_{2} M_{2} \\
& +A_{3} M_{3}+\frac{A_{5} M_{0}}{\Gamma(\beta+1)}+\left|\frac{\mu M_{0}}{(1+\mu) \Gamma(\alpha+\beta+1)}\right| .
\end{aligned}
$$

Then, $B_{r}$ is a closed, convex, and nonempty subset of the Banach space $X$.

Our aim is to prove that the operator $P$ has a unique fixed point on $B_{r}$. We show that $P B_{r} \subseteq B_{r}$.

For $x \in B_{r}, t \in[0,1]$, we have

$$
\begin{aligned}
& |P x(t)| \leq \frac{1}{\Gamma(\alpha+\beta)} \int_{0}^{t}(t-s)^{\alpha+\beta-1}\left|f\left(s, x(s), I^{p} x(s)\right)\right| d s \\
& +\frac{\lambda}{\Gamma(\alpha)} \int_{0}^{t}(t-s)^{\alpha-1}|x(s)| d s+\left|A_{1}(t)\right| \int_{0}^{1}|h(s ; x(s))| d s \\
& +\left|A_{2}(t)\right| \int_{0}^{1}|g(s ; x(s))| d s+\left|A_{3}(t)\right| \int_{0}^{1}|k(s ; x(s))| d s \\
& +\frac{\left|A_{4}(t)\right|}{\Gamma(\beta-\alpha)} \int_{0}^{1}(1-s)^{\beta-\alpha-1}\left|f\left(s, x(s), I^{P} x(s)\right)\right| d s \\
& +\frac{\left|A_{5}(t)\right|}{\Gamma(\beta)} \int_{0}^{1}(1-s)^{\beta-1}\left|f\left(s, x(s), I^{p} x(s)\right)\right| d s \\
& +\left|\frac{\mu \lambda}{(1+\mu) \Gamma(\alpha)}\right| \int_{0}^{1}(1-s)^{\alpha-1}|x(s)| d s \\
& +\left|\frac{\mu}{(1+\mu) \Gamma(\alpha+\beta)}\right| \int_{0}^{1}(1-s)^{\alpha+\beta-1}\left|f\left(s, x(s), I^{p} x(s)\right)\right| d s \\
& \leq \frac{1}{\Gamma(\alpha+\beta)} \int_{0}^{t}(t-s)^{\alpha+\beta-1}\left[\left|f\left(s, x(s), I^{p} x(s)\right)-f(s, 0,0)\right|\right. \\
& +|f(s, 0,0)|] d s+\frac{\lambda}{\Gamma(\alpha)} \int_{0}^{t}(t-s)^{\alpha-1}|x(s)| d s \\
& +\left|A_{1}(t)\right| \int_{0}^{1}|h(s ; x(s))-h(s ; 0)|+|h(s ; 0)| d s \\
& +\left|A_{2}(t)\right| \int_{0}^{1}|g(s ; x(s))-g(s ; 0)|+|g(s ; 0)| d s \\
& +\left|A_{3}(t)\right| \int_{0}^{1}|k(s ; x(s))-k(s ; 0)|+|k(s ; 0)| d s \\
& +\frac{\left|A_{4}(t)\right|}{\Gamma(\beta-\alpha)} \int_{0}^{1}(1-s)^{\beta-\alpha-1}\left[\mid f\left(s, x(s), I^{p} x(s)\right)\right. \\
& -f(s, 0,0)|+| f(s, 0,0) \mid] d s+\frac{\left|A_{5}(t)\right|}{\Gamma(\beta)} \int_{0}^{1}(1-s)^{\beta-1} \\
& \cdot\left[\left|f\left(s, x(s), I^{p} x(s)\right)-f(s, 0,0)\right|+|f(s, 0,0)|\right] d s \\
& +\left|\frac{\mu \lambda}{(1+\mu) \Gamma(\alpha)}\right| \int_{0}^{1}(1-s)^{\alpha-1}|x(s)| d s \\
& +\left|\frac{\mu}{(1+\mu) \Gamma(\alpha+\beta)}\right| \int_{0}^{1}(1-s)^{\alpha+\beta-1} \\
& \times\left[\left|f\left(s, x(s), I^{p} x(s)\right)-f(s, 0,0)\right|+|f(s, 0,0)|\right] d s,
\end{aligned}
$$

which implies that

$$
\begin{aligned}
\|P x\| \leq & \frac{1}{\Gamma(\alpha+\beta+1)}\left[\left(q_{1}+\frac{q_{2}}{\Gamma(p+1)}\right)\|x\|+M_{0}\right] \\
& +\frac{\lambda}{\Gamma(\alpha+1)}\|x\|+A_{1}\left[q_{4}\|x\|+M_{2}\right]+A_{2}\left[q_{3}\|x\|+M_{1}\right] \\
& +A_{3}\left[q_{5}\|x\|+M_{3}\right]+\frac{A_{4}}{\Gamma(\beta-\alpha+1)} \\
& \cdot\left[\left(q_{1}+\frac{q_{2}}{\Gamma(p+1)}\right)\|x\|+M_{0}\right]+\frac{A_{5}}{\Gamma(\beta+1)} \\
& \cdot\left[\left(q_{1}+\frac{q_{2}}{\Gamma(p+1)}\right)\|x\|+M_{0}\right]+\left|\frac{\mu \lambda}{(1+\mu) \Gamma(\alpha+1)}\right|\|x\| \\
& +\left|\frac{\mu}{(1+\mu) \Gamma(\alpha+\beta+1)}\right|\left[\left(q_{1}+\frac{q_{2}}{\Gamma(p+1)}\right)\|x\|+M_{0}\right] \\
\leq & {\left[( q _ { 1 } + \frac { q _ { 2 } } { \Gamma ( p + 1 ) } ) \left(\frac{1}{\Gamma(\alpha+\beta+1)}+\frac{A_{4}}{\Gamma(\beta-\alpha+1)}\right.\right.} \\
& \left.+\frac{A_{5}}{\Gamma(\beta+1)}+\left|\frac{\mu}{(1+\mu) \Gamma(\alpha+\beta+1)}\right|\right)+\frac{\lambda}{\Gamma(\alpha+1)} \\
& \left.+\left|\frac{\mu \lambda}{(1+\mu) \Gamma(\alpha+1)}\right|+A_{1} q_{4}+A_{2} q_{3}+A_{3} q_{5}\right]\|x\| \\
& +\frac{M_{0}}{\Gamma(\alpha+\beta+1)}+\frac{M_{0} A_{4}}{\Gamma(\beta-\alpha+1)}+A_{1} M_{2}+A_{2} M_{1}
\end{aligned}
$$

Now, for $x, y \in B_{r}$ and for $t \in[0 ; 1]$,

$$
\begin{aligned}
\mid P x(t) & -P y(t) \mid \\
\leq & \frac{1}{\Gamma(\alpha+\beta)} \int_{0}^{t}(t-s)^{\alpha+\beta-1} \mid f\left(s, x(s), I^{p} x(s)\right) \\
& -f\left(s, y(s), I^{p} y(s)\right)\left|d s+\frac{\lambda}{\Gamma(\alpha)} \int_{0}^{t}(t-s)^{\alpha-1}\right| x(s) \\
& -y(s)|d s+| A_{1}(t)\left|\int_{0}^{1}\right| h(s ; x(s))-h(s ; y(s)) \mid d s \\
& +\left|A_{2}(t)\right| \int_{0}^{1}|g(s ; x(s))-g(s ; y(s))| d s+\left|A_{3}(t)\right| \\
& \cdot \int_{0}^{1}|k(s ; x(s))-k(s ; y(s))| d s+\frac{\left|A_{4}(t)\right|}{\Gamma(\beta-\alpha)} \int_{0}^{1} \\
& \cdot(1-s)^{\alpha-\beta-1}\left|f\left(s, x(s), I^{p} x(s)\right)-f\left(s, y(s), I^{p} y(s)\right)\right| d s \\
& +\frac{\left|A_{5}(t)\right|}{\Gamma(\beta)} \int_{0}^{1}(1-s)^{\beta-1} \mid f\left(s, x(s), I^{p} x(s)\right) \\
& -f\left(s, y(s), I^{p} y(s)\right)|d s+| \frac{\mu \lambda}{(1+\mu) \Gamma(\alpha)} \mid \int_{0}^{1}(1-s)^{\alpha-1} \\
& \cdot|x(s)-y(s)| d s+\left|\frac{\mu}{(1+\mu) \Gamma(\alpha+\beta)}\right| \int_{0}^{1}(1-s)^{\alpha+\beta-1} \\
& \cdot\left|f\left(s, x(s), I^{p} x(s)\right)-f\left(s, y(s), I^{p} y(s)\right)\right| d s .
\end{aligned}
$$


Thus,

$$
\|P x-P y\| \leq r_{1}\|x-y\| .
$$

Since $r_{1}<1$, then operator $P$ is a contraction mapping. Therefore, boundary value problem (1) has a unique solution.

Theorem 7. Assume that $\left(H_{1}\right)$ and $\left(H_{2}\right)$ hold, $f:[0 ; 1] \times$ $\mathbb{R}^{2} \longrightarrow \mathbb{R}$ is a continuous function. Further, we suppose

$\left(H_{3}\right)-|f(t, x, y)| \leq m(t) ; \quad|h(t, x)| \leq \rho(t) ; \quad|k(t, x)| \leq \psi$ $(t) ;|g(t, x)| \leq \varphi(t), \forall(t, x, y) \in[0,1] \times \mathbb{R}^{2}$ with $m, \rho, \varphi, \psi \in C$ $\left([0,1] ; \mathbb{R}^{+}\right)$.Then, boundary value problem (1) has at least one solution on $[0,1]$ if $Q<1$ and $r_{1}^{\prime}<1$, where

$$
\begin{aligned}
Q= & {\left[A_{1} q_{4}+A_{2} q_{3}+A_{3} q_{5}+\frac{A_{4}}{\Gamma(\beta-\alpha+1)}\left(q_{1}+\frac{q_{2}}{\Gamma(p+1)}\right)\right.} \\
& +\frac{A_{5}}{\Gamma(\beta+1)}\left(q_{1}+\frac{q_{2}}{\Gamma(p+1)}\right)+\frac{|\mu \lambda|}{|(1+\mu)| \Gamma(\alpha+1)} \\
& \left.+\frac{|\mu|}{|(1+\mu)| \Gamma(\alpha+\beta+1)}\left(q_{1}+\frac{q_{2}}{\Gamma(p+1)}\right)\right], \\
r^{\prime}{ }_{1}= & \left|\frac{\lambda}{\Gamma(\alpha+1)}\right|+\frac{|\mu \lambda|}{(|1+\mu|) \Gamma(\alpha+1)} .
\end{aligned}
$$

Proof. Consider the closed ball $B_{r}{ }^{\prime}=\left\{x \in X:\|x\| \leq r^{\prime}\right\}$ with the radius $r^{\prime} \geq\left(r_{2}^{\prime} /\left(1-r_{1}^{\prime}\right)\right)$, where

$$
\begin{aligned}
r_{2}^{\prime}= & \frac{\|m\|}{\Gamma(\alpha+\beta+1)}+A_{1}\|\rho\|+A_{2}\|\phi\|+A_{3}\|\psi\| \\
& +\frac{A_{4}\|m\|}{\Gamma(\beta-\alpha+1)}+\frac{A_{5}\|m\|}{\Gamma(\beta+1)}+\frac{|\mu|\|m\|}{(|1+\mu|) \Gamma(\alpha+\beta+1)} .
\end{aligned}
$$

We introduce the decomposition $P=P_{1}+P_{2}$, where

$$
\begin{aligned}
P_{1} x(t)= & \frac{1}{\Gamma(\alpha+\beta)} \int_{0}^{t}(t-s)^{\alpha+\beta-1} f\left(s, x(s), I^{p} x(s)\right) d s \\
& -\frac{\lambda}{\Gamma(\alpha)} \int_{0}^{t}(t-s)^{\alpha-1} x(s) d s, \\
P_{2} x(t)= & A_{1}(t) \int_{0}^{1} h(s ; x(s)) d s+A_{2}(t) \int_{0}^{1} g(s ; x(s)) d s \\
& +A_{3}(t) \int_{0}^{1} k(s ; x(s)) d s+\frac{A_{4}(t)}{\Gamma(\beta-\alpha)} \int_{0}^{1} \\
& \cdot(1-s)^{\beta-\alpha-1} f\left(s, x(s), I^{p} x(s)\right) d s+\frac{A_{5}(t)}{\Gamma(\beta)} \int_{0}^{1} \\
& \cdot(1-s)^{\beta-1} f\left(s, x(s), I^{p} x(s)\right) d s+\frac{\mu \lambda}{(1+\mu) \Gamma(\alpha)} \\
& \cdot \int_{0}^{1}(1-s)^{\alpha-1} x(s) d s-\frac{\mu}{(1+\mu) \Gamma(\alpha+\beta)} \\
& \cdot \int_{0}^{1}(1-s)^{\alpha+\beta-1} f\left(s, x(s), I^{p} x(s)\right) d s .
\end{aligned}
$$

For $x, y \in B_{r}$, we have

$$
\begin{aligned}
& \left|P_{1} x(t)+P_{2} y(t)\right| \\
& \leq \frac{1}{\Gamma(\alpha+\beta)} \int_{0}^{t}(t-s)^{\alpha+\beta-1}\left|f\left(s, x(s), I^{P} x(s)\right)\right| d s \\
& +\left|\frac{\lambda}{\Gamma(\alpha)}\right| \int_{0}^{t}(t-s)^{\alpha-1}|x(s)| d s+\left|A_{1}(t)\right| \int_{0}^{1}|h(s ; y(s))| d s \\
& +\left|A_{2}(t)\right| \int_{0}^{1}|g(s ; y(s))| d s+\left|A_{3}(t)\right| \int_{0}^{1}|k(s ; y(s))| d s \\
& +\frac{\left|A_{4}(t)\right|}{\Gamma(\beta-\alpha)} \int_{0}^{1}(1-s)^{\beta-\alpha-1}\left|f\left(s, y(s), I^{p} y(s)\right)\right| d s \\
& +\frac{\left|A_{5}(t)\right|}{\Gamma(\beta)} \int_{0}^{1}(1-s)^{\beta-1}\left|f\left(s, y(s), I^{p} y(s)\right)\right| d s \\
& +\frac{|\mu \lambda|}{(|1+\mu|) \Gamma(\alpha)} \int_{0}^{1}(1-s)^{\alpha-1}|y(s)| d s \\
& +\frac{|\mu|}{(|1+\mu|) \Gamma(\alpha+\beta)} \int_{0}^{1}(1-s)^{\alpha+\beta-1}\left|f\left(s, y(s), I^{p} y(s)\right)\right| d s \\
& \leq \frac{1}{\Gamma(\alpha+\beta)} \int_{0}^{t}(t-s)^{\alpha+\beta-1} m(s) d s \\
& +\left|\frac{\lambda}{\Gamma(\alpha)}\right| \int_{0}^{t}(t-s)^{\alpha-1}|x(s)| d s+A_{1} \int_{0}^{1} \rho(s) d s \\
& +A_{2} \int_{0}^{1} \phi(s) d s+A_{3} \int_{0}^{1} \psi(s) d s+\frac{A_{4}}{\Gamma(\beta-\alpha)} \int_{0}^{1} \\
& \cdot(1-s)^{\beta-\alpha-1} m(s) d s+\frac{A_{5}}{\Gamma(\beta)} \int_{0}^{1}(1-s)^{\beta-1} m(s) d s \\
& +\frac{|\mu \lambda|}{(|1+\mu|) \Gamma(\alpha)} \int_{0}^{1}(1-s)^{\alpha-1}|y(s)| d s \\
& +\frac{|\mu|}{(|1+\mu|) \Gamma(\alpha+\beta)} \int_{0}^{1}(1-s)^{\alpha+\beta-1} m(s) d s,
\end{aligned}
$$

which implies that

$$
\begin{aligned}
\left\|P_{1} x+P_{2} y\right\| \leq & \frac{\|m\|}{\Gamma(\alpha+\beta+1)}+\left|\frac{\lambda}{\Gamma(\alpha+1)}\right| r^{\prime}+A_{1}\|\rho\| \\
& +A_{2}\|\phi\|+A_{3}\|\psi\|+\frac{A_{4}\|m\|}{\Gamma(\beta-\alpha+1)} \\
& +\frac{A_{5}\|m\|}{\Gamma(\beta+1)}+\frac{|\mu \lambda|}{(|1+\mu|) \Gamma(\alpha+1)} r^{\prime} \\
& +\frac{|\mu|\|m\|}{(|1+\mu|) \Gamma(\alpha+\beta+1)} \leq r^{\prime} .
\end{aligned}
$$


For $x, y \in B_{r}{ }^{\prime}$, we have

$$
\begin{aligned}
\mid P_{2} x( & t)-P_{2} y(t) \mid \\
\leq & \left.\left|A_{1}(t)\right| \int_{0}^{1} \mid h(s ; x(s))\right)-h(s ; y(s)) \mid d s \\
& +\left|A_{2}(t)\right| \int_{0}^{1}|g(s ; x(s))-g(s ; y(s))| d s \\
& +\left|A_{3}(t)\right| \int_{0}^{1}|k(s ; x(s))-k(s ; y(s))| d s \\
& +\frac{\left|A_{4}(t)\right|}{\Gamma(\beta-\alpha)} \int_{0}^{1}(1-s)^{\alpha-\beta-1} \mid f\left(s, x(s), I^{p} x(s)\right) \\
& -f\left(s, y(s), I^{p} y(s)\right) \mid d s+\frac{\left|A_{5}(t)\right|}{\Gamma(\beta)} \int_{0}^{1}(1-s)^{\beta-1} \\
& \cdot\left|f\left(s, x(s), I^{p} x(s)\right)-f\left(s, y(s), I^{p} y(s)\right)\right| d s \\
& +\frac{|\mu \lambda|}{|(1+\mu)| \Gamma(\alpha)} \int_{0}^{1}(1-s)^{\alpha-1}|x(s)-y(s)| d s \\
& +\frac{|\mu|}{|(1+\mu)| \Gamma(\alpha+\beta)} \int_{0}^{1}(1-s)^{\alpha+\beta-1} \\
& \cdot\left|f\left(s, x(s), I^{p} x(s)\right)-f\left(s, y(s), I^{p} y(s)\right)\right| d s \\
\leq & A_{1} \int_{0}^{1} q_{4}|x(s)-y(s)| d s+A_{2}\left|\int_{0}^{1} q_{3}\right| x(s)-y(s) \mid d s \\
& +A_{3} \int_{0}^{1} q_{5}|x(s)-y(s)| d s+\frac{A_{4}}{\Gamma(\beta-\alpha)} \int_{0}^{1} \\
& \cdot(1-s)^{\beta-\alpha-1}\left(q_{1}|x(s)-y(s)|+q_{2}\left|I^{p} x(s)-I^{p} y(s)\right|\right) d s \\
& +\frac{A_{5}}{\Gamma(\beta)} \int_{0}^{1}(1-s)^{\beta-1}\left(q_{1}|x(s)-y(s)|\right. \\
& \cdot(1-s)^{\alpha+\beta-1}\left(I_{1} x(s)-I^{p} y(s) \mid\right) d s+\frac{|\mu \lambda|}{|(1+\mu)| \Gamma(\alpha)} \int_{0}^{1} \\
& \left(1-1|x(s)-y(s)| d s+\frac{|\mu|}{|(1+\mu)| \Gamma(\alpha+\beta)} \int_{0}^{1}\right. \\
& \\
& \left(1-y(s)\left|+q_{2}\right| I^{p} x(s)-I^{p} y(s) \mid\right) d s, \\
&
\end{aligned}
$$

which implies that

$$
\begin{aligned}
\left\|P_{2} x-P_{2} y\right\| \leq & A_{1} q_{4}\|x-y\|+A_{2} q_{3}\|x-y\|+A_{3} q_{5}\|x-y\| \\
& +\frac{A_{4}}{\Gamma(\beta-\alpha+1)}\left(q_{1}+\frac{q_{2}}{\Gamma(p+1)}\right)\|x-y\| \\
& +\frac{A_{5}}{\Gamma(\beta+1)}\left(q_{1}+\frac{q_{2}}{\Gamma(p+1)}\right)\|x-y\| \\
& +\frac{|\mu \lambda|}{|(1+\mu)| \Gamma(\alpha+1)}\|x-y\|+\frac{|\mu|}{|(1+\mu)| \Gamma(\alpha+\beta+1)} \\
& \cdot\left(q_{1}+\frac{q_{2}}{\Gamma(p+1)}\right)\|x-y\| \leq\left[A_{1} q_{4}+A_{2} q_{3}+A_{3} q_{5}\right.
\end{aligned}
$$

$$
\begin{aligned}
& +\frac{A_{4}}{\Gamma(\beta-\alpha+1)}\left(q_{1}+\frac{q_{2}}{\Gamma(p+1)}\right)+\frac{A_{5}}{\Gamma(\beta+1)} \\
& \cdot\left(q_{1}+\frac{q_{2}}{\Gamma(p+1)}\right)+\frac{|\mu \lambda|}{|(1+\mu)| \Gamma(\alpha+1)} \\
& \left.+\frac{|\mu|}{|(1+\mu)| \Gamma(\alpha+\beta+1)}\left(q_{1}+\frac{q_{2}}{\Gamma(p+1)}\right)\right]\|x-y\| \\
& \leq Q\|x-y\|,
\end{aligned}
$$

since $Q<1$, then $P_{2}$ is a contraction.

Next, we show that $P_{1}$ is compact and continuous. Continuity of $f$ implies that the operator $P_{1}$ is continuous.

Since, $\quad\left\|P_{1} x\right\| \leq(\|m\| /(\Gamma(\alpha+\beta+1)))+|(\lambda /(\Gamma(\alpha+1)))|$ $r^{\prime}$, therefore, $P_{1}$ is uniformly bounded on $B_{r}{ }^{\prime}$. Suppose that $0 \leq t_{1}<t_{2} \leq 1$. We have

$$
\begin{aligned}
\left|P_{1} x\left(t_{2}\right)-P_{1} x\left(t_{1}\right)\right| & \\
\leq & \frac{1}{\Gamma(\alpha+\beta)} \mid \int_{0}^{t_{1}}\left(\left(t_{2}-s\right)^{\alpha+\beta-1}-\left(t_{1}-s\right)^{\alpha+\beta-1}\right) f \\
& \cdot\left(s, x(s), I^{p} x(s)\right) d s+\int_{t_{1}}^{t_{2}}\left(t_{2}-s\right)^{\alpha+\beta-1} f\left(s, x(s), I^{p} x(s)\right) d s \mid \\
\leq & +\frac{|\lambda|}{\Gamma(\alpha)} \mid \int_{0}^{t_{1}}\left(\left(t_{2}-s\right)^{\alpha-1}-\left(t_{1}-s\right)^{\alpha-1}\right) x(s) d s \\
& +\int_{t_{1}}^{t_{2}}\left(t_{2}-s\right)^{\alpha-1} x(s) d s \mid \frac{\|m\|}{\Gamma(\alpha+\beta+1)}\left[2\left(t_{2}-t_{1}\right)^{\alpha+\beta}\right. \\
& \left.+\left|t_{1}^{\alpha+\beta}-t_{2}^{\alpha+\beta}\right|\right]+\frac{|\lambda| r}{\Gamma(\alpha+1)}\left[2\left(t_{2}-t_{1}\right)^{\alpha}+\left|t_{1}^{\alpha}-t_{2}^{\alpha}\right|\right] .
\end{aligned}
$$

As $t_{1} \rightarrow t_{2}$, the above expression tends to be zero independently from $x \in B_{r}{ }^{\prime}$. This implies that $P_{1}$ is relatively compact on $B_{r}{ }^{\prime}$. Then, by the Arzela-Ascoli theorem, operator $P_{1}$ is compact on $B_{r}{ }^{\prime}$.

Therefore, according to the Krasnoselskii fixed point theorem, problem (1) has at least one solution on $B_{r}{ }^{\prime}$.

\section{Example}

Example 8. Consider the following boundary value problem:

$$
\left\{\begin{array}{l}
{ }^{c} D^{3 / 4}\left({ }^{c} D^{1 / 3}+\frac{1}{300}\right) x(t)=\frac{1}{500+t^{2}}\left(\sin x+\frac{1}{\Gamma(3 / 4)} \int_{0}^{t}(t-s)^{-1 / 4} x d s\right), \quad t \in[0,1] \\
x(0)+x(1)=\frac{1}{200} \int_{0}^{1} \frac{|x(s)|}{300+|x(s)|} d s \\
{ }^{c} D^{1 / 3} x(0)+{ }^{c} D^{1 / 3} x(1)=\frac{1}{200} \int_{0}^{1}\left(\frac{1}{s+2}\right)^{3} \frac{|x(s)|}{30+|x(s)|} d s \\
{ }^{c} D^{2 / 3} x(0)+{ }^{c} D^{2 / 3} x(1)=\frac{1}{200} \int_{0}^{1}\left(\frac{1}{s+4}\right)^{2} \frac{|x(s)|}{30+|x(s)|} d s .
\end{array}\right.
$$


We choose $\beta=4 / 3, \alpha=1 / 3, \lambda=1 / 300, \mu=1, \sigma_{1}=1 / 200$, $\sigma_{2}=1 / 200, \sigma_{3}=1 / 200, p=1 / 4$, and the continuous functions

$$
\begin{aligned}
f\left(t, x, I^{1 / 4} x\right) & =\frac{1}{500+t^{2}}\left(\sin x+\frac{1}{\Gamma(3 / 4)} \int_{0}^{t}(t-s)^{-1 / 4} x d s\right), \\
g(t, x) & =\frac{|x(t)|}{300+|x(t)|}, \\
h(t, x) & =\left(\frac{1}{t+2}\right)^{3} \frac{|x(t)|}{30+|x(t)|},
\end{aligned}
$$

$$
k(t, x)=\left(\frac{1}{t+4}\right)^{2} \frac{|x(t)|}{30+|x(t)|} .
$$
$1 / 480$,

Clearly, $q_{1}=q_{2}=1 / 500, q_{3}=1 / 300, q_{4}=1 / 240$, and $q_{5}=$

Then, we have $r_{1} \approx 0.07<1$.

Thus, all the assumptions of Theorem 2 hold. Then, problem (29) has a unique solution.

Example 9. Consider the following boundary value problem:

$$
\left\{\begin{array}{l}
{ }^{c} D^{3 / 4}\left({ }^{c} D^{1 / 3}+\frac{1}{300}\right) x(t)=\frac{1}{(t+10)^{2}}\left(\frac{1}{5+x^{2}}+\int_{0}^{t}(t-s)^{-1 / 4} \frac{1}{5+x^{2}} d s\right), \quad t \in[0,1], \\
x(0)+x(1)=\frac{1}{200} \int_{0}^{1} \frac{1}{s+10} \frac{1}{30+|x(s)|} d s, \\
{ }^{c} D^{1 / 3} x(0)+{ }^{c} D^{1 / 3} x(1)=\frac{1}{200} \int_{0}^{1}\left(\frac{1}{s+2}\right)^{3} \frac{|x(s)|}{30+|x(s)|} d s \\
{ }^{c} D^{2 / 3} x(0)+{ }^{c} D^{2 / 3} x(1)=\frac{1}{200} \int_{0}^{1}\left(\frac{1}{s+4}\right)^{2} \frac{|x(s)|}{30+|x(s)|} d s .
\end{array}\right.
$$

Here, $\beta=3 / 4, \alpha=1 / 3, \lambda=1 / 30, \mu=1, \sigma_{1}=1 / 200, \sigma_{2}=1$ $1200, \sigma_{3}=1 / 200, p=1 / 4$, and the continuous functions

$$
\begin{aligned}
f\left(t, x, I^{1 / 4} x\right) & =\frac{1}{(t+10)^{2}}\left(\frac{1}{5+x^{2}}+\int_{0}^{t}(t-s)^{-1 / 4} \frac{1}{5+x^{2}} d s\right), \\
g(t, x) & =\frac{1}{t+10} \frac{|x(t)|}{30+|x(t)|}, \\
h(t, x) & =\left(\frac{1}{t+2}\right)^{3} \frac{|x(t)|}{30+|x(t)|}, \\
k(t, x) & =\left(\frac{1}{t+4}\right)^{2} \frac{|x(t)|}{30+|x(t)|} .
\end{aligned}
$$

Clearly, $q_{1}=q_{2}=1 / 500, q_{3}=1 / 300, q_{4}=1 / 240$, and $q_{5}=$ $1 / 480$; thus, we have $Q \approx 0.057<1$ and $r^{\prime}{ }_{1} \approx 0,0065<1$.

Then, problem (31) has a least one solution.

\section{Conclusion}

In this paper, we studied the existence and uniqueness of fractional Langevin equations with nonseparated integral boundary conditions. First of all, we transformed the problem into an equivalent fixed point problem; second of all, we utilized the Banach contraction principle and the Krasnoselskii fixed point theorem to prove the existence and uniqueness of solutions.

\section{Data Availability}

No data were used to support this study.

\section{Conflicts of Interest}

The authors declare that there are no conflicts of interest regarding the publication of this paper.

\section{Authors' Contributions}

All authors contributed equally to the writing of this paper. All authors read and approved the final manuscript.

\section{References}

[1] V. Lakshmikantham, "Theory of fractional functional differential equations," Nonlinear Analysis: Theory, Methods \& Applications, vol. 69, no. 10, pp. 3337-3343, 2008.

[2] V. Lakshmikantham and A. S. Vatsala, "Basic theory of fractional differential equations," Nonlinear Analysis: Theory, Methods \& Applications, vol. 69, no. 8, pp. 2677-2682, 2008.

[3] R. Hilfer, Applications of Fractional Calculs in Physics, World Scientific, Singapore, 2000.

[4] K. S. Miller and B. Ross, An Introduction to the Fractional Calculus and Fractional Differential Equations, Wiley, New York, NY, USA, 1993.

[5] I. Podlubny, Fractional Differential Equations, Academic Press, New York, NY, USA, 1993. 
[6] Y. Zhou, Basic Theory of Fractional Differential Equations, Xiangtan University, China, 2014.

[7] A. A. Kilbas, H. M. Srivastava, and J. J. Trujillo, Theory and Applications of Fractional Differential Equations, NorthHolland Mathematics Studies, vol. 204, Elsevier, Amsterdam, 2006.

[8] K. Hilal, L. Ibnelazyz, K. Guida, and S. Melliani, "Existence of mild solutions for an impulsive fractional integro-differential equations with non-local condition," in Recent Advances in Intuitionistic Fuzzy Logic Systems, S. Melliani and O. Castillo, Eds., vol. 372 of Studies in Fuzziness and Soft Computing, , pp. 251-271, Springer, Cham, 2019.

[9] K. Hilal, K. Guida, L. Ibnelazyz, and M. Oukessou, "Existence results for an impulsive fractional integro-differential equations with a non-compact semigroup," in Recent Advances in Intuitionistic Fuzzy Logic Systems, S. Melliani and O. Castillo, Eds., vol. 372 of Studies in Fuzziness and Soft Computing, , pp. 191-211, Springer, Cham, 2019.

[10] Z. Zhou and Y. Qiao, "Solutions for a class of fractional Langevin equations with integral and anti-periodic boundary conditions," Boundary Value Problems, vol. 2018, no. 1, 2018.

[11] A. Salem and B. Alghamdi, "Multi-strip and multi-point boundary conditions for fractional Langevin equation," Fractal and Fractional, vol. 4, no. 2, p. 18, 2020.

[12] A. Salem and B. Alghamdi, "Multi-point and anti-periodic conditions for generalized Langevin equation with two fractional orders," Fractal and Fractional, vol. 3, no. 4, p. 51, 2019.

[13] H. Fazli, H. G. Sun, and J. J. Nieto, "Fractional Langevin equation involving two fractional orders: existence and uniqueness revisited," Mathematics, vol. 8, no. 5, p. 743, 2020.

[14] H. Fazli and J. J. Nieto, "Fractional Langevin equation with anti-periodic boundary conditions," Chaos, Solitons and Fractals, vol. 114, pp. 332-337, 2018.

[15] R. Zwanzig, Nonequilibrium Statistical Mechanics, Oxford University Press, Oxford, UK, 2001.

[16] W. T. Coffey, Y. P. Kalmykov, and J. T. Waldron, The Langevin Equation, World Scientific, Singapore, 2nd edition, 2004.

[17] F. Mainardi and P. Pironi, "The fractional Langevin equation: Brownian motion revisited," Extracta Math, vol. 10, pp. 140154, 1996.

[18] J. W. Bruce, "Fractal physiology and the fractional calculus: a perspective," Frontiers in Physiology, vol. 1, p. 12, 2010.

[19] B. Ahmad and J. J. Nieto, "Solvability of nonlinear Langevin equation involving two fractional orders with Dirichlet boundary conditions," International Journal of Differential Equations, vol. 2010, Article ID 649486, 10 pages, 2010.

[20] B. Ahmad, J. J. Nieto, and A. Alsaedi, "A nonlocal three-point inclusion problem of Langevin equation with two different fractional orders," Advances in Difference Equations, vol. 2012, no. 1, Article ID 54, 2012.

[21] J. R. Wang, S. Peng, and D. ORegan, "Local stable manifold of Langevin differential equations with two fractional derivatives," Advances in Difference Equations, vol. 2017, Article ID 355, 2017.

[22] G. Wang, L. Zhang, and G. Song, "Boundary value problem of a nonlinear Langevin equation with two different fractional orders and impulses," Fixed Point Theory and Applications, vol. 2012, Article ID 200, 2012.

[23] T. Yu, K. Deng, and M. Luo, "Existence and uniqueness of solutions of initial value problems for nonlinear Langevin equation involving two fractional orders," Communications in Nonlinear Science and Numerical Simulation, vol. 19, no. 6, pp. 1661-1668, 2014.

[24] C. Zhai, P. Li, and H. Li, "Single upper-solution or lowersolution method for Langevin equations with two fractional orders," Advances in Difference Equations, vol. 2018, no. 1, Article ID 360, 2018.

[25] C. Zhai and P. Li, "Nonnegative solutions of initial value problems for Langevin equations involving two fractional orders," Mediterranean Journal of Mathematics, vol. 15, no. 4, article 164, 2018.

[26] B. Ahmad, J. J. Nieto, A. Alsaedi, and M. EI-Shahed, "A study of nonlinear Langevin equation involving two fractional orders in different intervals," Nonlinear Analysis: Real World Applications, vol. 13, no. 2, pp. 599-606, 2012.

[27] B. Ahmad, A. Alsaedi, and S. Salem, "On a nonlocal integral boundary value problem of nonlinear Langevin equation with different fractional orders," Advances in Difference Equations, vol. 2019, no. 1, Article ID 57, 2019.

[28] A. Salem, F. Alzahrani, and L. Almaghamsi, "Fractional Langevin equations with nonlocal integral boundary conditions," Mathematics, vol. 7, no. 5, p. 402, 2019.

[29] B. Ahmad, J. J. Nieto, and A. Alsaedi, "Existence and uniqueness of solutions for nonlinear fractional differential equations with non-separated type integral boundary conditions," Acta Mathematica Scientia, vol. 31, no. 6, pp. 2122-2130, 2011.

[30] A. Krasnoselskii, "Two remarks on the method of successive approximations," Uspekhi Matematicheskikh Nauk, vol. 10, pp. 123-127, 1955. 